\title{
Critical care for concomitant severe traumatic brain injury and acute spinal cord injury in the polytrauma patient: illustrative case
}

\author{
Hansen Deng, MD, ${ }^{1}$ Diego D. Luy, BS, ${ }^{1}$ Hussam Abou-Al-Shaar, MD, ${ }^{1}$ John K. Yue, MD, ${ }^{2}$ Pascal O. Zinn, MD, ${ }^{1}$ \\ Ava M. Puccio, RN, PhD, ${ }^{1,3}$ and David O. Okonkwo, MD, PhD ${ }^{1,3}$ \\ ${ }^{1}$ Department of Neurological Surgery and ${ }^{3}$ Brain Trauma Research Center, University of Pittsburgh Medical Center, Pittsburgh, Pennsylvania; and ${ }^{2}$ Department of Neurological \\ Surgery, University of California, San Francisco, San Francisco, California
}

\begin{abstract}
BACKGROUND The occurrence of traumatic brain injury with spinal cord injury (SCl) in polytrauma patients is associated with significant morbidity. Clinicians face challenges from a decision-making and rehabilitative perspective. Management is complex and understudied. Treatment should be systematic beginning at the scene, focusing on airway resuscitation and hemodynamic stabilization, immobilization, and timely transport. Early operative interventions should be provided, followed by minimizing secondary pathophysiology. The authors present a case to delineate decisionmaking in the treatment of combined cranial and spinal trauma.

OBSERVATIONS A 19-year-old man presented as a level I trauma patient after falling 30 feet as the result of scaffolding collapse. The patient was unresponsive and was intubated; he had an initial Glasgow Coma Scale score of 4. Computed tomography revealed multicompartmental bleeding and herniation, for which supra- and infratentorial decompressive craniectomies were performed. The patient also suffered from thoracic $\mathrm{SCl}$ that resulted in complete paraplegia. Multimodality monitoring was used. After stabilization and lengthy rehabilitation, the patient obtained significant functional improvement.

LESSONS The approach to initial management of concomitant head and spine trauma is to establish intracranial stability followed by intraspinal stability. Patients can make considerable recovery, particularly younger patients, who are more likely to benefit from early aggressive interventions and medical treatment.
\end{abstract}

https://thejns.org/doi/abs/10.3171/CASE21521

KEYWORDS traumatic brain injury; spinal cord injury; polytrauma; decompressive hemicraniectomy

The annual incidence of traumatic brain injury (TBI) is between 5,000 and 8,000 cases per million, ${ }^{1}$ and the incidence of traumatic spinal cord injury (SCl) is 40 cases per million in the United States. $^{2}$ Dual diagnosis of TBI and $\mathrm{SCl}$ is less common at 3 to 10 cases per million, ${ }^{3}$ but it leads to increased morbidity and reduced overall quality of life. The current TBI models of prognostication are not specific to this subpopulation of patients. ${ }^{4}$ In part due to the complexity of specialized care and availability of medical resources, early management strategies of combined neuroaxis injury vary among providers and institutions.

Management of the combination of craniospinal trauma begins at the scene of the injury. Key points include resuscitation, early intubation and ventilation, spinal immobilization, and rapid patient transport to the nearest level I trauma center. Computed tomography $(\mathrm{CT})$ is first-line imaging for initial cranial and spinal bony evaluation. The goal of early operative decompression is to prevent neurological deterioration and obtain mechanical stability. Invasive monitoring at bedside can further reduce secondary insults after initial trauma. ${ }^{5}$

Neurological recovery from $\mathrm{SCl}$ depends on completeness of lesion with respect to sacral sparing. ${ }^{6}$ The American Spinal Injury Association Impairment Scale (AIS) is the gold standard for injury classification by muscle groups and sensory points. ${ }^{7}$ Patients with concurrent TBI and $\mathrm{SCl}$ have longer length of stay and rehabilitation, and the trajectory of

ABBREVIATIONS AIS = American Spinal Injury Association Impairment Scale; CT = computed tomography; EVD = external ventricular drain; GCS = Glasgow Coma Scale; ICP = intracranial pressure; $\mathrm{LD}=$ lumbar drain; $\mathrm{OR}=$ operating room; $\mathrm{SCl}=$ spinal cord injury; TBI = traumatic brain injury.

INCLUDE WHEN CITING Published January 10, 2022; DOI: 10.3171/CASE21521.

SUBMITTED September 15, 2021. ACCEPTED November 8, 2021.

(C) 2022 The authors, CC BY-NC-ND 4.0 (http://creativecommons.org/licenses/by-nc-nd/4.0/). 

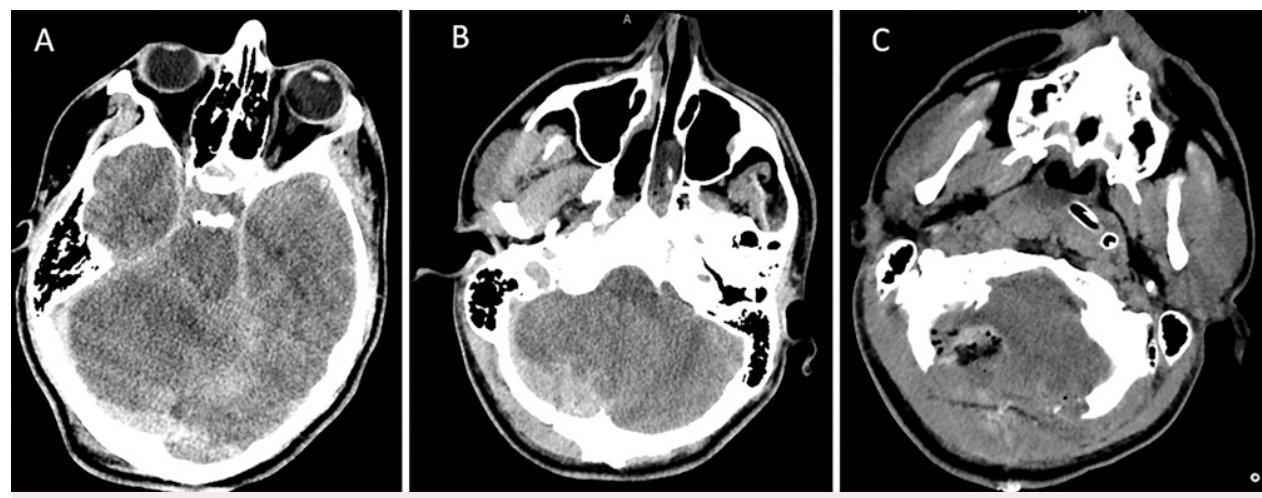

FIG. 1. CT of the head on presentation with infratentorial injury. A: Infratentorial edema on the initial imaging demonstrating effacement of suprasellar cisterns with mass effect on the brainstem and radiographic evidence of upward transtentorial herniation. B: Bilateral occipital fractures with right subdural hematoma. C: Postoperative suboccipital craniectomy and subdural evacuation with decreased mass effect.

neurological recovery after discharge is not well understood. We present the clinical decision-making process for a patient who sustained complex severe TBI and SCl, in whom early stepwise operative decompressions and stabilization, along with multimodality monitoring, assisted with overall improvement in neurological function.

\section{Illustrative Case}

A 19-year-old male construction worker with no significant past medical history presented as a level I trauma patient after falling 30 feet from scaffolding along with his coworker, who died at the scene. The patient was intubated and had an initial Glasgow Come Scale (GCS) score of 4 (eye [E] 1; verbal [V] 1; motor [M] 2). Pupils were reactive, but gag and cough reflexes were not detected.

CT of the head showed extensive bilateral occipital fractures, infratentorial swelling, and right subdural hematoma causing upward transtentorial herniation (Fig. 1A). The patient also had supratentorial multicompartmental bleeding with bifrontal subdural hematoma and diffuse traumatic subarachnoid hemorrhage (Fig. 2). CT of the thoracic spine revealed a three-column fracture dislocation of the T9 and T10 levels, T10 burst fracture with retropulsion, and severe spinal stenosis (Fig. 3A). Other injuries included left pneumothorax, rib fractures, and pleural effusion and lung contusion.

The patient was taken to the operating room (OR) for emergency suboccipital decompressive craniectomy, subdural evacuation, and external ventricular drain (EVD) placement for intracranial pressure (ICP) monitoring. The EVD opening pressure was $20 \mathrm{~mm}$ Hg. Postoperative CT demonstrated blossoming bifrontal contusions with associated mass effect (Fig. 1C). The patient returned to the operating room for Kjellberg bifrontal decompressive craniectomy and subdural evacuation. ${ }^{8}$ He was stabilized in the neurotrauma intensive care unit. A lumbar drain (LD) was placed to assist with titration of spinal perfusion pressure of $65 \mathrm{~mm} \mathrm{Hg}$. On hospital day 3 with improved ICPs, the patient returned to the OR for his SCl, including T7-T12 posterior spinal fusion for definitive fixation and T9-T10 decompression (Fig. 2B).

By hospital day 3, neurological examination improved to GCS score 8 (E2V1M5). His mental status continued to improve to GCS 10 (E4V1M5) by day 6 . On day 8, the patient began to follow commands in upper extremities, resulting in GCS 11 (E4V1M6). His SCl on the AIS was incomplete grade B with sacral sparing. Tracheostomy was performed on hospital day 14. The extended hospital course included the treatment of Staphylococcus epidermidis meningitis, ventilator-associated pneumonia, and sacral decubitus ulcers. Cranioplasty was performed while he was an inpatient, and on hospital day 50, the patient was discharged to specialized $\mathrm{SCl}$ rehabilitation. He completed a 6week course of comprehensive therapy, including daily physical therapy, occupational therapy, and speech and language pathology.

Six months postinjury, the patient returned to follow-up accompanied by his girlfriend. Functional status on the Glasgow Outcome Scale Extended was $6{ }^{9} \mathrm{He}$ was cognitively near baseline and fully conversant with his tracheostomy decannulated. The patient used a wheelchair and exhibited 0/5 strength in his bilateral lower extremities, but he started working as a furniture maker in a woodworking shop.

\section{Discussion}

\section{Observations}

The comorbidities of patients having concurrent TBI and $\mathrm{SCl}$ have been described in the trauma literature, ${ }^{10,11}$ but determining the best management strategies remains clinically challenging and underreported. Limited studies on incidence of dual diagnosis of TBI with SCl can range from $16 \%$ to $60 \%$, with most patients having
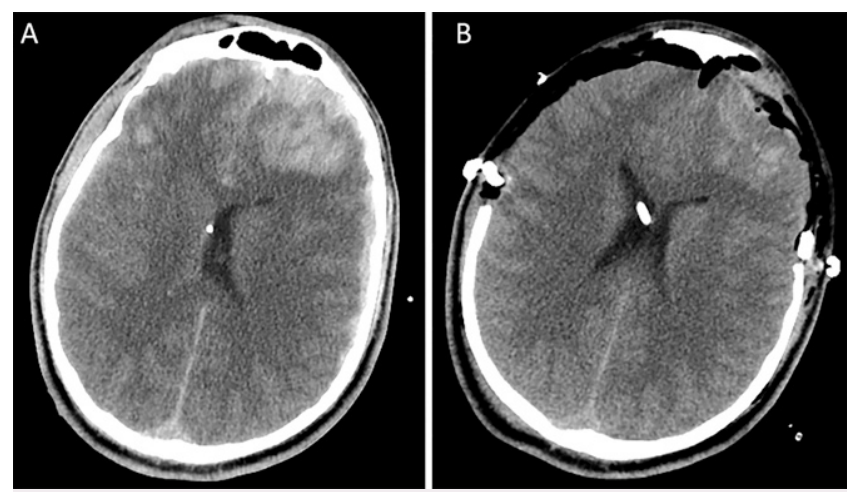

FIG. 2. CT of the head on presentation with supratentorial injury. A: Preoperative axial CT image displaying blossoming bifrontal contusions with associated mass effect after suboccipital decompressive craniectomy. B: Postoperative axial CT demonstrating Kjellberg bifrontal decompressive craniectomy and subdural evacuation. 

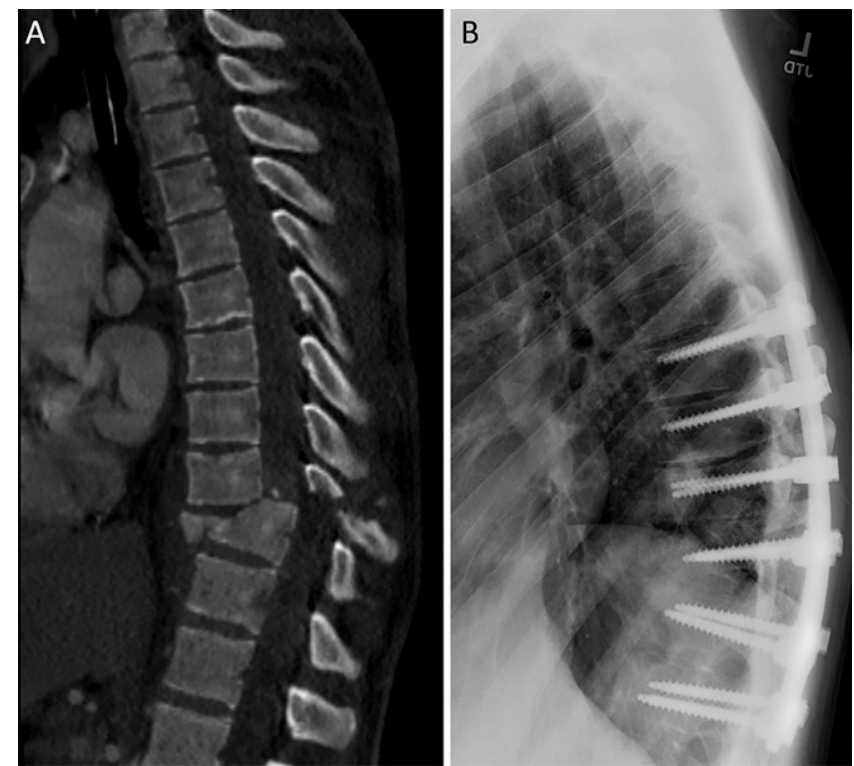

FIG. 3. CT of the spine on presentation. A: Three-column fracture dislocation of the T9 and T10 levels, T10 burst fracture with retropulsion, and severe spinal stenosis. B: Postoperative sagittal CT after T7-T12 posterior spinal fusion for definitive fixation and T9-T10 decompression.

sustained mild-to-moderate TBI. ${ }^{3,12}$ Currently, the treatment paradigm of combined $\mathrm{TBI}$ and $\mathrm{SCl}$ is highly variable and an evolving specialized field. Additionally, understanding of the interaction of neuroaxis polytrauma during recovery is lacking. To our knowledge, this is the first documented case of a patient with severe TBI necessitating supraand infratentorial decompressive craniectomies who, despite having sustained a significant $\mathrm{SCl}$, has made a favorable recovery.

In the acute period after initial trauma, treatment of intracranial dysfunction takes precedence over spinal cord pathology. However, operative decompression for $\mathrm{SCl}$ should not be delayed once a patient can tolerate surgery from an intracranial perspective. ${ }^{13}$ In the present case, craniectomy was more prioritized over spinal decompression in the emergency setting of severe TBI. Spinal cord perfusion was optimized while awaiting sufficient patient stability for return to the $\mathrm{OR}$ for spinal decompression. Measures to prevent secondary pathophysiology of intracranial and spinal cord insults can be pursued in parallel at the bedside. Strategies include multimodality intracranial monitoring and treatment of ICP and brain tissue oxygenation using tiered treatment algorithms. ${ }^{14}$ Growing evidence supports LD placement to monitor intrathecal pressure and titrate spinal cord perfusion to minimize hypoperfusion. ${ }^{15}$

Accurate prognostication after combined severe $\mathrm{TBI}$ and $\mathrm{SCl}$ is difficult, and the overall long-term recovery of survivors is not well known. Neurological improvement can be further delayed by the increased risks of nosocomial infection, the need for mechanical ventilation due to respiratory insufficiency, and effects of autonomic dysregulation. As observed here, a patient with concurrent TBI and $\mathrm{SCl}$ requires extended hospitalization and rehabilitation, but there is potential for considerable progress toward regaining functional independence through multidisciplinary support. Particularly in the younger population, early prognosis of dual diagnosis of $\mathrm{TBI}$ and $\mathrm{SCl}$ should be approached by the clinician with caution.

\section{Lessons}

Critical care for the polytraumatic patient with severe head and spinal trauma places emphasis on accurate assessment of injury complex and systematic stabilization of the neuroaxis. Early operative interventions should be performed for intracranial pathology followed by intraspinal pathology, as dictated in our institutional practice. Secondary insults occur across a more protracted phase and require imperative attention and correction. Early prognostication should be approached cautiously, especially because knowledge of predictors of recovery in this patient population remains lacking.

\section{Acknowledgments}

We acknowledge Peter C. Gerszten, MD, and Amir Faraji, MD, for their clinical care and input.

\section{References}

1. Rutland-Brown W, Langlois JA, Thomas KE, Xi YL. Incidence of traumatic brain injury in the United States, 2003. J Head Trauma Rehabil. 2006;21(6):544-548.

2. Yue JK, Winkler EA, Rick JW, et al. Update on critical care for acute spinal cord injury in the setting of polytrauma. Neurosurg Focus. 2017;43(5):E19.

3. Hagen EM, Eide GE, Rekand T, Gilhus NE, Gronning M. Traumatic spinal cord injury and concomitant brain injury: a cohort study. Acta Neurol Scand Suppl. 2010;(190):51-57.

4. Roozenbeek B, Lingsma HF, Lecky FE, et al. Prediction of outcome after moderate and severe traumatic brain injury: external validation of the International Mission on Prognosis and Analysis of Clinical Trials (IMPACT) and Corticoid Randomisation After Significant Head injury (CRASH) prognostic models. Crit Care Med. 2012;40(5): 1609-1617.

5. Carney N, Totten AM, O'Reilly C, et al. Guidelines for the Management of Severe Traumatic Brain Injury, Fourth Edition. Neurosurgery. 2017;80(1):6-15.

6. Chay W, Kirshblum S. Predicting outcomes after spinal cord injury. Phys Med Rehabil Clin N Am. 2020;31(3):331-343.

7. Roberts TT, Leonard GR, Cepela DJ. Classifications in brief: American Spinal Injury Association (ASIA) impairment scale. Clin Orthop Relat Res. 2017;475(5):1499-1504.

8. Kjellberg RN, Prieto A Jr. Bifrontal decompressive craniotomy for massive cerebral edema. J Neurosurg. 1971;34(4):488-493.

9. Wilson JTL, Pettigrew LE, Teasdale GM, Teasdale GM. Structured interviews for the Glasgow Outcome Scale and the extended Glasgow Outcome Scale: guidelines for their use. J Neurotrauma. 1998;15(8):573-585.

10. Macciocchi SN, Bowman B, Coker J, Apple D, Leslie D. Effect of co-morbid traumatic brain injury on functional outcome of persons with spinal cord injuries. Am J Phys Med Rehabil. 2004;83(1): 22-26.

11. Bradbury CL, Wodchis WP, Mikulis DJ, et al. Traumatic brain injury in patients with traumatic spinal cord injury: clinical and economic consequences. Arch Phys Med Rehabil. 2008;89(12 suppl): S77-S84.

12. Macciocchi S, Seel RT, Thompson N, Byams R, Bowman B. Spinal cord injury and co-occurring traumatic brain injury: assessment and incidence. Arch Phys Med Rehabil. 2008;89(7):1350-1357.

13. Burke JF, Yue JK, Ngwenya LB, et al. Ultra-early ( $<12$ hours) surgery correlates with higher rate of American Spinal Injury Association impairment scale conversion after cervical spinal cord injury. Neurosurgery. 2019;85(2):199-203.

14. Okonkwo DO, Shutter LA, Moore C, et al. Brain oxygen optimization in severe traumatic brain injury phase-II: a phase II randomized trial. Crit Care Med. 2017;45(11):1907-1914. 
15. Kwon BK, Curt A, Belanger LM, et al. Intrathecal pressure monitoring and cerebrospinal fluid drainage in acute spinal cord injury: a prospective randomized trial. J Neurosurg Spine. 2009;10(3): 181-193.

\section{Disclosures}

The authors report no conflict of interest concerning the materials or methods used in this study or the findings specified in this paper.

\section{Author Contributions}

Conception and design: Deng, Luy, Abou-Al-Shaar, Yue, Puccio,

Okonkwo. Acquisition of data: Deng, Luy, Abou-Al-Shaar, Zinn, Puccio,
Okonkwo. Analysis and interpretation of data: Deng, Luy, Abou-AlShaar, Yue, Okonkwo. Drafting the article: Deng, Luy, Yue, Puccio, Okonkwo. Critically revising the article: Abou-Al-Shaar, Yue, Zinn, Puccio, Okonkwo. Reviewed submitted version of manuscript: Abou-AlShaar, Yue, Zinn, Puccio, Okonkwo. Statistical analysis: Okonkwo. Administrative/technical/material support: Okonkwo. Study supervision: Puccio, Okonkwo. Operating surgeon: Zinn.

\section{Correspondence}

Hansen Deng: University of Pittsburgh Medical Center, Pittsburgh, PA. dengh3@upmc.edu. 